\title{
Enhanced flow of core-softened fluids through narrow nanotubes
}

Cite as: J. Chem. Phys. 140, 194504 (2014); https://doi.org/10.1063/1.4876555

Submitted: 28 November 2013 . Accepted: 30 April 2014 . Published Online: 21 May 2014

José Rafael Bordin, José S. Andrade, Alexandre Diehl, and Marcia C. Barbosa
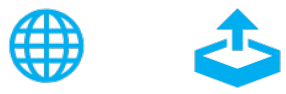

\section{ARTICLES YOU MAY BE INTERESTED IN}

Diffusion enhancement in core-softened fluid confined in nanotubes

The Journal of Chemical Physics 137, 084504 (2012); https://doi.org/10.1063/1.4746748

How fast does water flow in carbon nanotubes?

The Journal of Chemical Physics 138, 094701 (2013); https://doi.org/10.1063/1.4793396

Structure and mobility of water confined in $\mathrm{AlPO}_{4}-54$ nanotubes

The Journal of Chemical Physics 146, 234509 (2017); https://doi.org/10.1063/1.4985626

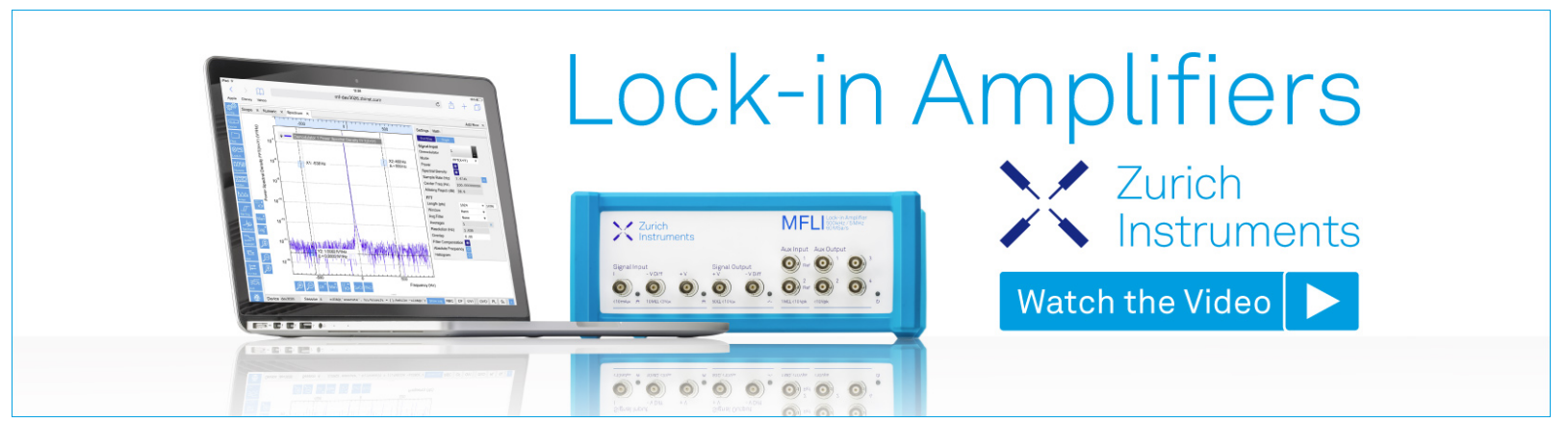

J. Chem. Phys. 140, 194504 (2014); https://doi.org/10.1063/1.4876555 


\title{
Enhanced flow of core-softened fluids through narrow nanotubes
}

\author{
José Rafael Bordin, ${ }^{1,2, a)}$ José S. Andrade, Jr., ${ }^{3, b)}$ Alexandre Diehl, ${ }^{2, c)}$ \\ and Marcia C. Barbosa ${ }^{4, d)}$ \\ ${ }^{1}$ Campus Caçapava do Sul, Universidade Federal do Pampa, Av. Pedro Anunciação, s/n ${ }^{0}$, CEP 96570-000, \\ Caçapava do Sul, RS, Brazil \\ ${ }^{2}$ Departamento de Física, Instituto de Física e Matemática, Universidade Federal de Pelotas, \\ Caixa Postal 354, CEP 96010-900, Pelotas, RS, Brazil \\ ${ }^{3}$ Departamento de Física, Universidade Federal do Ceará, CEP 60451-970, Fortaleza, Ceará, Brazil \\ ${ }^{4}$ Instituto de Física, Universidade Federal do Rio Grande do Sul, Caixa Postal 15051, CEP 91501-970, \\ Porto Alegre, RS, Brazil
}

(Received 28 November 2013; accepted 30 April 2014; published online 21 May 2014)

\begin{abstract}
We investigate through non-equilibrium molecular dynamic simulations the flow of anomalous fluids inside rigid nanotubes. Our results reveal an anomalous increase of the overall mass flux for nanotubes with sufficiently smaller radii. This is explained in terms of a transition from a single-file type of flow to the movement of an ordered-like fluid as the nanotube radius increases. The occurrence of a global minimum in the mass flux at this transition reflects the competition between the two characteristic length scales of the core-softened potential. Moreover, by increasing further the radius, another substantial change in the flow behavior, which becomes more evident at low temperatures, leads to a local minimum in the overall mass flux. Microscopically, this second transition is originated by the formation of a double-layer of flowing particles in the confined nanotube space. These nano-fluidic features give insights about the behavior of confined isotropic anomalous fluids. @ 2014 AIP Publishing LLC. [http://dx.doi.org/10.1063/1.4876555]
\end{abstract}

\section{INTRODUCTION}

The recent development of nanofabrication techniques have opened the possibility of building nanoscale structures ${ }^{1}$ that can effectively mimic biological channels. ${ }^{2}$ This new technology has generated devices with application not only as controlled models for biological systems, but also as laboratory gatekeepers that are chemically selective and could therefore eventually function as nanofilters. ${ }^{3}$ In this way, the understanding of the nanofluidic inside this devices is of great interest.

Some fluids, even in bulk, exhibit an anomalous behavior, distinct from the observed in most liquids. While the majority of the liquids contract on cooling there is some anomalous liquids in with a maximum in the density is observed at constant pressure. Also, unlike the general behavior, some liquid diffusion coefficients increase under compression. These anomalous fluids include water, ${ }^{4-6} \mathrm{Te},{ }^{7} \mathrm{Ga}, \mathrm{Bi},{ }^{8} \mathrm{Si},{ }^{9}, 10 \mathrm{Ge}_{15} \mathrm{Te}_{85},{ }^{11}$ liquid metals, ${ }^{12}$ and graphite. ${ }^{13}$ Computational studies for silica, ${ }^{14-16}$ silicon, ${ }^{17}$ and $\mathrm{BeF}_{2}{ }^{14}$ also indicate the presence of thermodynamic anomalies for these materials. ${ }^{6}$ In addition, silica, ${ }^{15-18}$ silicon, ${ }^{19}$ and water ${ }^{20,21}$ also have a maximum in the diffusion coefficient at constant pressure.

At the nanoscale, however, fluid properties differ significantly from what usual hydrodynamics would predict. Most of the recent experiments about confined anomalous fluids are related to water, but a few analysis have been done in

\footnotetext{
a) josebordin@unipampa.edu.br

b) soares@ fisica.ufc.br

c) diehl@ufpel.edu.br

d) marcia.barbosa@ufrgs.br
}

alkanes $^{22}$ where a rapid but not as fast as the flux observed in water was also measured. For instance, recent experiments highlight the exceptional properties of carbon nanotubes. ${ }^{23-25}$ Precisely, these studies revealed that certain microelectromechanical fabrication processes are capable to assemble a macroscopic collection of carbon nanotubes with diameters in a range as small as 1.3 to $2 \mathrm{~nm}$. It has been shown that the water flux in these special membranes can be three to four orders of magnitude larger than the value prediction from the continuum-based no-slip Hagen-Poiseuille (HP) relation, ${ }^{24,26}$ as also confirmed by computer simulations. ${ }^{27}$ This water flow increase is not uniform with the different nanotube radius. As the nanotube radius is reduced, the flow decreases up to a certain threshold, and for smaller radius it increases again, as reported by computational ${ }^{27}$ and experimental ${ }^{25}$ works. This non-monotonic behavior is attributed to the transition from continuum to sub-continuum transport as the nanotube shrinks ${ }^{25,27}$ and can also be driven by hydrophobicity. ${ }^{28}$ However, despite the use of all atom models for water, the computational results are only qualitatively comparable to the experiments. The approximations employed in the water models and friction effects might be the origin of these numerical differences. ${ }^{29}$ Also, the evaluation of the slip length of confined water seems to be sensible to the employed computational method, and non-equilibrium Molecular Dynamics (NEMD) simulations can lead to spurious results. ${ }^{30,31}$ The different atomistic models for water give, therefore, a qualitative description of the experimental enhancement flow. This suggests that the major ingredient for the rapid flux is not related to the details of the model, but to the effective interactions that they do represent. 
In the last two decades, a number of effective model potentials have been suggested for anomalous fluids based on water. ${ }^{32-36}$ Despite their simplicity, these models exhibit in bulk the thermodynamic, dynamic, and structural anomalies of water and other liquids, and also predict the existence of a second critical point hypothesized by Poole $e t$ al. for the ST2 water model..$^{37}$ In these models, the particle-particle interaction is modeled through a potential with two characteristic length scales, that is, one repulsive shoulder and an attractive well. ${ }^{36,38-40}$ The competition between these two scales leads to the density and diffusion anomalies. Likewise, other interesting systems, such as colloids and globular proteins, can be modeled using two length scales potential. ${ }^{41,42}$

Recently, it has been suggested that core-softened potential models are able to capture some of the anomalous behavior even under confinement. ${ }^{43-47}$ In fact, the behavior of the diffusion constant for nanoconfined water, attributed to the hydrogen bonds, ${ }^{48}$ was obtained by pure volumetric effects in these isotropic anomalous fluids. ${ }^{46}$ Motivated by this proposition, in this paper we use NEMD simulation in order to explore the connection between the enhanced particle flow and the fluid-fluid interaction potential for fluids confined inside a nanopore. In order to avoid misleadings, we should address that in the literature, ${ }^{25,27,47}$ it is usual to evaluate the flow enhancement factor, which can be defined as the ratio between the hydraulic conductivity obtained using molecular simulations and the hydraulic conductivity predicted by classical continuum models. In our paper, the enhanced flow is the anomalous increase of particle flow in narrow nanotubes, and will not be compared with the results of classical continuum theories. Distinct soft-core fluids are tested, such as one that exhibit anomalous properties ${ }^{36}$ and the standard LennardJones fluid. The same NEMD model was used recently by us in the context of ionic channels ${ }^{49}$ and core-softened fluids inside nanoporous. ${ }^{47} \mathrm{We}$ analyze only two length scales potential systems and therefore any comparison with the effects observed in alkanes where the fast flow is also observed should be taken with caution.

The paper is organized as follows. The model and the computational details are given in Sec. II. The results are discussed in Sec. III, and the summary and conclusions are presented in Sec. IV.

\section{THE MODEL SYSTEM AND THE SIMULATION METHODOLOGY}

The simulation setup, used in this work to obtain the dynamical behavior of anomalous fluid inside nanotubes, is essentially the same of our previous work, applied to the study of ionic fluxes through narrow nanopores and transmembrane channels ${ }^{49}$ as shown schematically in Fig. 1 . The simulation box is a cubic parallelepiped with dimensions $4 L \times L \times L$ in $x y z$ directions, containing two reservoirs (two control volumes (CVs), $\mathrm{CV}_{1}$ and $\mathrm{CV}_{2}$, where the chemical potential of the fluid is maintained fixed) and a nanotube connected to both CVs through small buffer regions. These two buffer regions (see Fig. 1) were used in order to avoid any influence of the simulation technique applied inside the CVs on the fluid dynamics through the nanotube. The nanotube structure is built

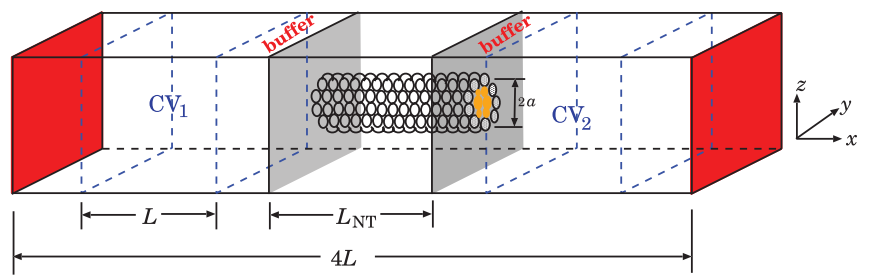

FIG. 1. Schematic depiction of the simulation box. The nanotube is built as a cylindrical tube, with radius $a$ and length $L_{\mathrm{NT}}$, made of a wrapped sheet of fixed Lennard-Jones (LJ) spheres. The system is confined in $x$ direction by two flat walls (red planes) at each end of the simulation cell. The same flat walls are used at both entrances (gray planes), except for the orifices, in order to maintain the flux between the two reservoirs (CVs) only through the nanotube. Two small buffer regions were included between both entrances of the nanotube and the two CVs. Periodic boundary conditions are applied only in $y$ and $z$ directions.

as a cylindrical tube with radius $a$ and length $L_{\mathrm{NT}}$, made of a wrapped sheet of stationary Lennard-Jones (LJ) particles with effective diameter $\sigma$. Both entrances of the nanotube, except for the orifices, are bounded by confining flat walls, as well as the two extremes of the simulation box in the $x$ direction, in order to maintain the movement of fluid particles between the two CVs only through the nanotube, according Fig. 1. With this setup, periodic boundary conditions are applied only in the $y$ and $z$ directions. We should address that the nanotube coarse-grained model used in our simulations is rougher than the surface of a carbon nanotube (CNT) but not as rough to change thermodynamic and dynamic properties. Also, the nanopore flexibility, with proper thermostatted walls, influences the dynamical, thermodynamical, and structural behavior of the confined fluid, ${ }^{50,51}$ and distinct results are obtained when the nanopore is rigid or flexible. ${ }^{44,52}$ Nevertheless, the difference between the results in non-equilibrium simulations with frozen or thermostatted walls are monotonic, ${ }^{50,51}$ and a qualitative comparison between the cases is valid. Therefore, our model reflects the case of rigid confinement inside a solid nanopore, and the results should be considered in this scenario.

The anomalous fluid system is modeled as a collection of spherical particles with effective diameter $\sigma$, the same of the nanotube particles, and mass $m$, interacting through the three dimensional core-softened potential ${ }^{36}$

$$
\begin{aligned}
U\left(r_{i j}\right)= & 4 \varepsilon\left[\left(\frac{\sigma}{r_{i j}}\right)^{12}-\left(\frac{\sigma}{r_{i j}}\right)^{6}\right] \\
& +u_{0} \varepsilon \exp \left[-\frac{1}{c^{2}}\left(\frac{r_{i j}-r_{0}}{\sigma}\right)^{2}\right],
\end{aligned}
$$

where $r_{i j}=\left|\vec{r}_{i}-\vec{r}_{j}\right|$ is the center-to-center distance between two fluid particles. The first term on the right is the standard 12-6 LJ potential, ${ }^{53}$ while the second term corresponds to a Gaussian centered at $r_{0}$, with depth $u_{0} \varepsilon$ and width $c \sigma$. For $u_{0}$ $=5.0, c=1.0$, and $r_{0}=0.7 \sigma$, the potential (1) displays two different length scales: one at $r_{i j} \approx \sigma$, where the force has a local minimum, and another at $r_{i j} \approx 2 \sigma$, where the fraction of imaginary modes shows a local maximum. ${ }^{54}$ We assume that the fluid-fluid interaction, Eq. (1), has a cutoff radius of $3.5 \sigma$ for all cases simulated. It has been previously observed ${ }^{36}$ 
that the pressure-temperature phase diagram of this system at equilibrium exhibits features similar to the anomalies present in water. ${ }^{6,55}$

The interaction between a fixed nanotube and an anomalous fluid particle, separated by a center-to-center distance $r$, is given by the purely repulsive Weeks-Chandler-Andersen (WCA) LJ potential ${ }^{53}$

$$
U^{\mathrm{WCA}}(r)= \begin{cases}U_{\mathrm{LJ}}(r)-U_{\mathrm{LJ}}\left(r_{c}\right), & r \leq r_{c}, \\ 0, & r>r_{c},\end{cases}
$$

where $U_{\mathrm{LJ}}(r)$ is the standard 12-6 LJ potential and $r_{c}=2^{1 / 6} \sigma$ is a cutoff distance. The confining flat walls are modeled with the same WCA LJ potential, however considering the $x$-projection of the distance between one fluid particle (outside the nanotube) and the wall position. The nanotube radius is varied from $a=1.3 \sigma$ to $a=9.0 \sigma$, while for the simulation box in $y$ and $z$ directions we have used two sizes: $L=10 \sigma$ for nanotubes with radius $a \leq 4 \sigma$ and $L=2 a+2 \sigma$ otherwise. Since in our model the nanotube particles have an effective diameter of $\sigma$, the available radius for fluid movement inside the nanotube is approximately $a-\sigma / 2$ in Fig. 1 .

A steady state flux of particles through the nanotube is induced by using the Dual Control Volume Grand Canonical Molecular Dynamics (DCV-GCMD) method. ${ }^{56}$ Briefly stated, in the DCV-GCMD simulations two CVs are initially prepared at desired densities, using Grand Canonical Monte Carlo (GCMC) simulations in both CVs, during $5 \times 10^{5}$ steps, with the initial velocity for each particle obtained from a Maxwell-Boltzmann distribution at the desired temperature. After that, the system evolves in time using the Molecular Dynamics (MD) methodology applied in all simulation box and, as a consequence of this dynamics, the densities in both CVs are changed. In order to restore the densities to their initial values, the MD steps are intercalated with insertion/deletion GCMC steps performed only inside the reservoirs. In our simulations we have used $150 \mathrm{GCMC}$ steps for every $500 \mathrm{MD}$ steps during the DCV-GCMD methodology, which ensures that the densities in both reservoirs change less than $2 \%$. Using this procedure, we have maintained fixed the densities in both CVs as $\rho_{1}=0.2 \sigma^{3}$ and $\rho_{2}=0.01 \sigma^{3}$ in $\mathrm{CV}_{1}$ and $\mathrm{CV}_{2}$, respectively. It should be noted that the use of small buffer regions between the reservoirs and the nanotube ends is essential to avoid any artificial effect in the vicinity of the nanotube entrances, produced by the GCMC steps inside the reservoirs. Since in these buffer regions particles evolve by MD integration, a particle inserted during a GCMC step that enters the buffer region reaches the right velocity distribution in few steps, before it eventually gets inside the nanotube. As a consequence, the flux steady state is obtained after $5 \times 10^{5} \mathrm{DCV}$ GCMD steps, after that the desired observables were typically obtained during $5 \times 10^{7}$ DCV-GCMD steps for each simulation, averaging over 10 to 20 independent runs. A constant time step of $\delta t=0.005$, in LJ time units,${ }^{53}$ is adopted. The average temperature of the system is fixed by means of the Nose-Hoover heat-bath scheme with a coupling parameter $Q$ $=2$. For simplicity, we assume that the nanotube atoms are motionless during the entire simulation. This is a valid approximation for the case of rigid nanoconfinement. However, we should address that the nanopore flexibility can lead to distinct results. $^{30,44}$ Therefore, since the nanotube particles are not time integrated, the thermostat is applied only to the fluid particles. The reduced temperature in our simulations is defined as $T^{*} \equiv k_{B} T / \varepsilon$, where $k_{B}$ is the Boltzmann constant.

The axial flux of particles through the tube, $J_{x \text {,tube }}$, is computed by counting the number of particles that cross the nanotube from left to right, $n_{\mathrm{ltr}}$, and the particles flowing from right to left, $n_{\mathrm{rtt}},{ }^{56}$

$$
J_{x, \text { tube }}=\frac{n_{\mathrm{ltr}}-n_{\mathrm{rtl}}}{A_{\mathrm{NT}} N_{\mathrm{steps}} \delta t},
$$

where $A_{\mathrm{NT}}=\pi a_{\mathrm{eff}}^{2}$, with $a_{\mathrm{eff}}=a-\sigma / 2$ the effective radius available for the fluid, $N_{\text {steps }}$ is the total number of steps used in the simulation, and $\delta t$ is the MD time step. In an entirely similar way, we evaluate the flux in the $x$-direction for the non-confined case, $J_{x \text {, bulk }}$, but now using $A=L \times L$, since there is no nanotube in the system.

\section{RESULTS AND DISCUSSION}

In order to characterize the effect of the two length scales present in the potential (1), simulations were performed for $u_{0}=5.0,3.0,1.0$ and $u_{0}=0$, which corresponds to the standard 12-6 LJ fluid, for a reduced temperature of $T^{*}=1.0$. In the first two cases, $u_{0}=5.0$ and 3.0, there is a competition between the two length scales, since the energetic penalty to move from one scale to another is high, as shown in the inset of Fig. 2. When applied in bulk conditions, these two cases will exhibit anomalous behavior. ${ }^{36}$ For the last two cases, on the other hand, there is no significant energetic barrier between the two length scales for $u_{0}=1.0$, and only one length scale (the minimum in the LJ potential) for $u_{0}=0$. Hence, in bulk conditions the fluid behavior is non anomalous for these low $u_{0}$ values. Our goal here is to check if the presence of anomalous behavior in bulk leads to the observed enhanced flow inside narrow nanotubes.

Figure 2 illustrates the dependence of the mass flux with the nanotube radius for different values of $u_{0}$. The inset of Fig. 2 shows how this parameter affects the shape of the

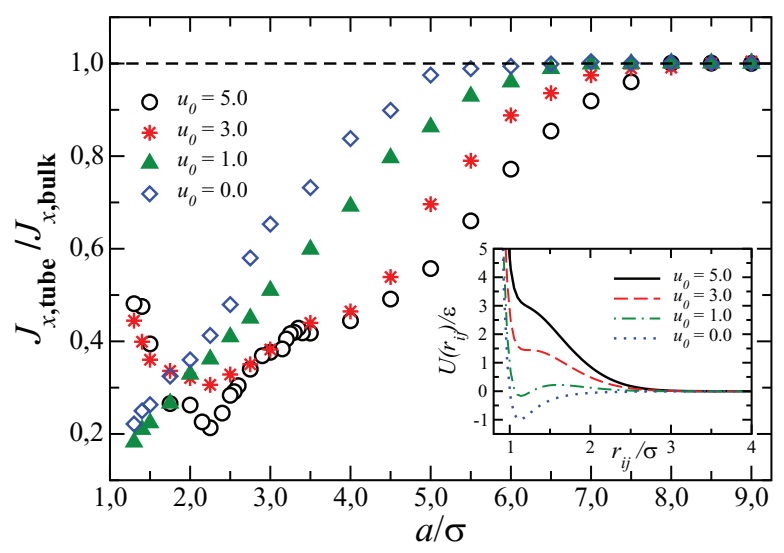

FIG. 2. Flux of particles through the nanotube, $J_{x}$,tube, in units of the nonconfined flux, $J_{x}$, bulk, for different interaction potential parameter $u_{0}$. The inset shows the fluid-fluid interaction, Eq. (1), as a function of the separation distance $r_{i j}=\left|\vec{r}_{i}-\vec{r}_{j}\right|$ between two particles for different values of $u_{0}$. 
interaction potential. For $u_{0}=5.0$ and sufficiently large values of the nanotube radius the flux reaches a maximum, which is approximately equals to the corresponding bulk value. As expected, by decreasing the radius, the flux gradually decreases from this saturation value, due to the obvious mass transport limitations of the confined nanotube geometry. Interestingly, as the radius is reduced even more, the flux reaches a minimum at a critical radius $a_{c} \approx 2.25 \sigma$ and starts to increase sharply, revealing anomalously large values for a range of significantly reduced nanotube radii. Anomalies in the transport behavior have also been previously observed with atomistic ${ }^{57}$ and effective models ${ }^{46}$ of water. In these cases, the diffusion coefficient is evaluated in confined environments, but under equilibrium conditions, which leads to an apparent measure. This can be substantially different from the effective mass transport $J_{x, \text { tube }}$ quantified here.

As shown in the inset of Fig. 2, the interaction potential for $u_{0}=3.0$ displays a shoulder-like profile with a small barrier between the two length scales. Our simulations for this case also indicate the presence of a minimum followed by anomalously large flux values for radii smaller than the critical radius $a_{c}$, as shown in Fig. 2. Moreover, despite of the decrease in the energy barrier from $u_{0}=5.0$ to $u_{0}=3.0$, the critical radius $a_{c}$ is essentially the same for both potentials, since they still possess identical characteristic lengths.

For $u_{0}=1.0$, the potential exhibits a quasi-LJ shape, as shown in the inset of the Fig. 2, with a small attractive well in the first characteristic length and a small barrier with a second characteristic length. As shown in Fig. 2, the anomalous behavior vanishes completely in this case. The competition between the two length scales becomes irrelevant as it becomes energetically viable for a particle to transit from positions corresponding to the first and second characteristic lengths. Previous works for this soft-core model shows that, even in bulk, this fluid only features anomalous behavior when exists competition between the length scales in the interaction potential. ${ }^{36}$ Finally, for the LJ fluid $\left(u_{0}=0\right.$ case) we also do not observe the anomalous increase in flux for small radius, as shown in Fig. 2, which is the expected behavior for simple LJ fluids. ${ }^{58}$ These results show that the presence of the two length scales are essential to observe an anomalous enhanced flow inside nanotubes. Therefore, the behavior can be explained in the framework of the competition between the two length scales potential and the confinement.

Despite the mathematical simplicity of the effective potential representing the fluid in our model, it is capable to catch several structural changes of the fluid, going from single-file flow in nanotubes with small radius, to bulklike flow in nanotubes with large radius. Between these two regimes, several other structures have been found, including ring structures, as shown in Fig. 3. As can be seen, there is a formation of structured layers inside the nanotube.

We can understand the dynamical behavior of the system, Fig. 2, through its underlying structural properties. Here, we consider typically the simulation results obtained with $u_{0}$ $=5.0$ at temperature $T^{*}=1.0$. Fluids modeled with twolength scales potential tend to display an ordered structure in bulk, which corresponds to the situation shown in Fig. 4(a), where the confining nanotube has a large radius, $a=4.0 \sigma$.
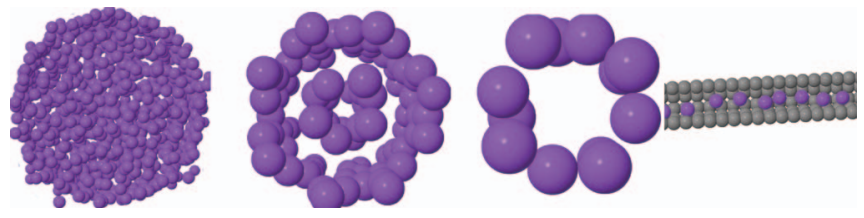

FIG. 3. Snapshot of the simulation box. The nanotube radii, from the left to the right, are $a=10 \sigma, a=4.5 \sigma, a=2.25 \sigma$, and $a=1.5 \sigma$. For better visualization, only for the case $a=1.5 \sigma$ the nanotube particles are shown.

The ordered behavior and the resulting high flux reflect the quasi-discrete nature of the particle flow. The distance between two adjacent density peaks is approximately equals to $\sigma$, the first length scale of the core-softened potential. The fluid therefore experiences an ordered liquid behavior, with a well defined structure and a high mobility, due to the changes in relative positions of interacting particles from one density peak to another. The highly ordered density profile of the fluid observed in Fig. 4(b) close to the critical nanotube radius $a_{c}$ is responsible for the low particle flux. This solid-like state, with a well defined structure and the minimum in the mobility, appears as a special condition due to a combined action of confinement and the presence of two length scales in the potential. As shown in Fig. 4(c), ordering disappears under extreme confinement to generate a single-file flow of enhanced flux, where the particles are obliged to remain at the first length scale.

We can obtain more details about the flux increase in anomalous fluids analyzing the radial velocity profile $v_{x, \mathrm{NT}}$. This profile shows the average velocity per particle as function of the Euclidian distance from the $x$ axis in cylindrical coordinates $r=\left(y^{2}+z^{2}\right)^{1 / 2}$. In Fig. 5(a) we show the velocity profile for the anomalous fluid, $u_{0}=5.0$. For nanotubes with radius $a=3.5 \sigma$, where the fluid is structured in a ring layer near the wall and a linear layer in the center, the velocity profile indicates that the central layer is moving faster than the external layer. This behavior is explained by the friction with the rough surface of the nanotube. At the critical radius,

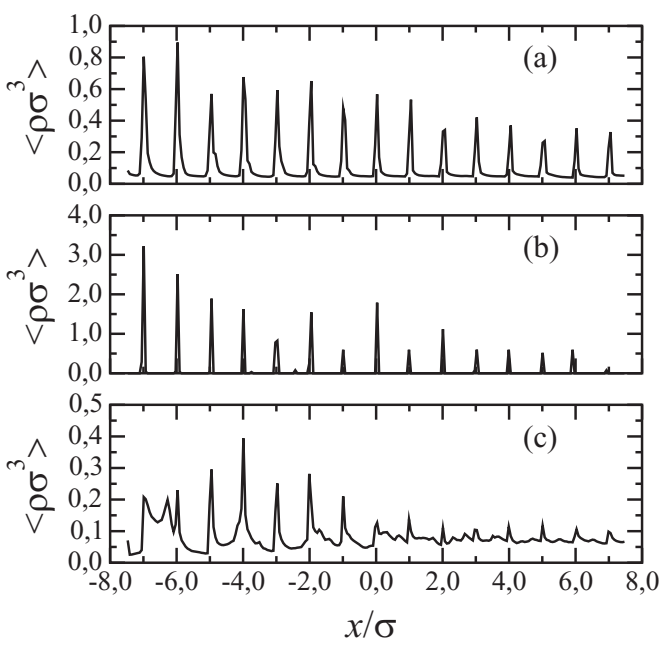

FIG. 4. Axial density profiles inside the nanotube for a fluid modeled with $u_{0}=5.0$ at temperature $T^{*}=1.0$. (a) An ordered liquid behavior is observed for a nanotube with $a=4.5 \sigma$, (b) a solid-like behavior for $a=2.0 \sigma$, and (c) a disordered structure appears for $a=1.25 \sigma$. 

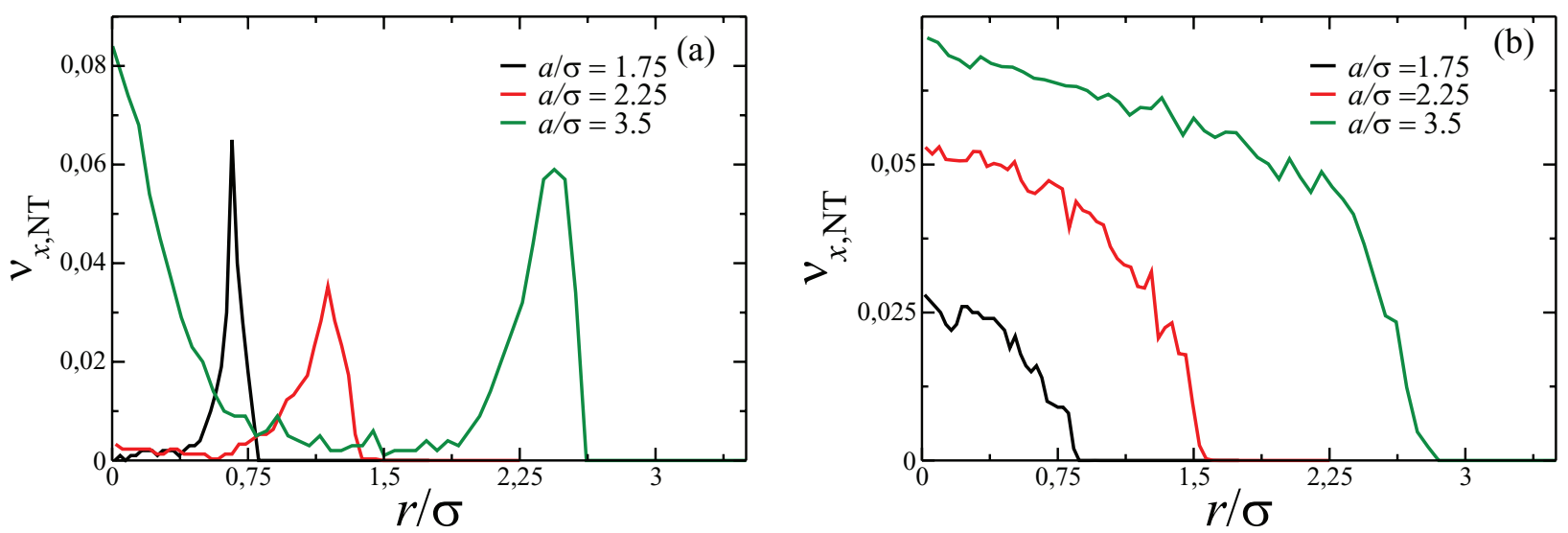

FIG. 5. Radial velocity profile for some values of nanotube radius for the case with (a) $u_{0}=5.0$ and (b) $u_{0}=0.0$.

$a_{c}=2.25 \sigma$, the fluid shows a ring structure in contact with the wall nanotube, as depicted in Fig. 3. The fluid-nanotube friction, allied to the particle distance equals to the second length scale, makes the fluid to move with a small velocity through the nanotube. However, when the nanotube radius is smaller than $a_{c}$, such as $a=1.75 \sigma$, the fluid assumes a single line structure and flows with a higher velocity. This occurs because the distance between the particles is no longer the second length scale. Also, the small nanotube radius leads the fluid particle to interact with more nanotube particles that surround it, unlike for the ring structure case, when the fluid only interacts with less nanotube particles. Nevertheless, we can see that the peak in the velocity profile is higher for $a$ $=1.75 \sigma$ than for $a=2.25 \sigma$. On the other hand, for the $\mathrm{LJ}$ fluid $\left(u_{0}=0.0\right.$ case $)$ we do not observe any structure. As a consequence, the velocity profile is uniform, as shown in Fig. 5(b). Also, as we decrease the nanotube radius, the velocity per particle decreases as well, resulting in flow decreasing. This result shows that the competition between the two length scales is crucial to the formation of layers, and it is strongly connected to the anomalous flow behavior.

Finally, we investigate the fluid flow behavior in the nanotube for $u_{0}=5.0$ at different temperatures. A similar behavior was observed, with the minimum flux located at the same value of critical radius $a_{c}$, as shown in Fig 6 for

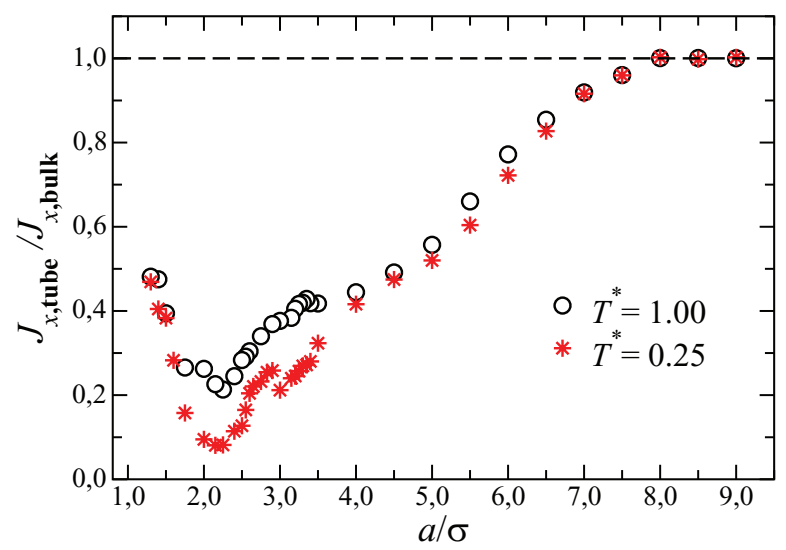

FIG. 6. Particle flux through the nanotube for the $u_{0}=5.0$ case, at different values of temperature.
$T^{*}=1.0$ and 0.25 . Surprisingly, a second anomalous behavior is revealed at lower temperatures. The system at $T^{*}=0.25$ shows an anomalous flux increase in the region $2.75 \sigma \leq a$ $\leq 3.0 \sigma$. Looking in detail the curve for $T^{*}=1.0$, the same behavior can be observed in the region $3.12 \sigma \leq a \leq 3.5 \sigma$. This anomaly occurs due to changes in the fluid structure from a double layer of flowing particles (a cylindrical layer near the nanotube wall and a linear central layer) to a single cylindrical layer structure. For nanotubes with radius $a$ $=3.5 \sigma$, the system displays the same double layer structure for both temperatures, as shown in Fig. 7(a). For $a=3.25 \sigma$ the single layer structure appears at $T^{*}=1.0$, while at a lower temperature $T^{*}=0.25$ the fluid still flows through a double layer structure, as depicted in Fig. 7(b). This change in the fluid conformation causes the observed flux anomaly, being a consequence of the competition between the fluid-fluid and the fluid-nanotube interactions. The fluid particles tend to
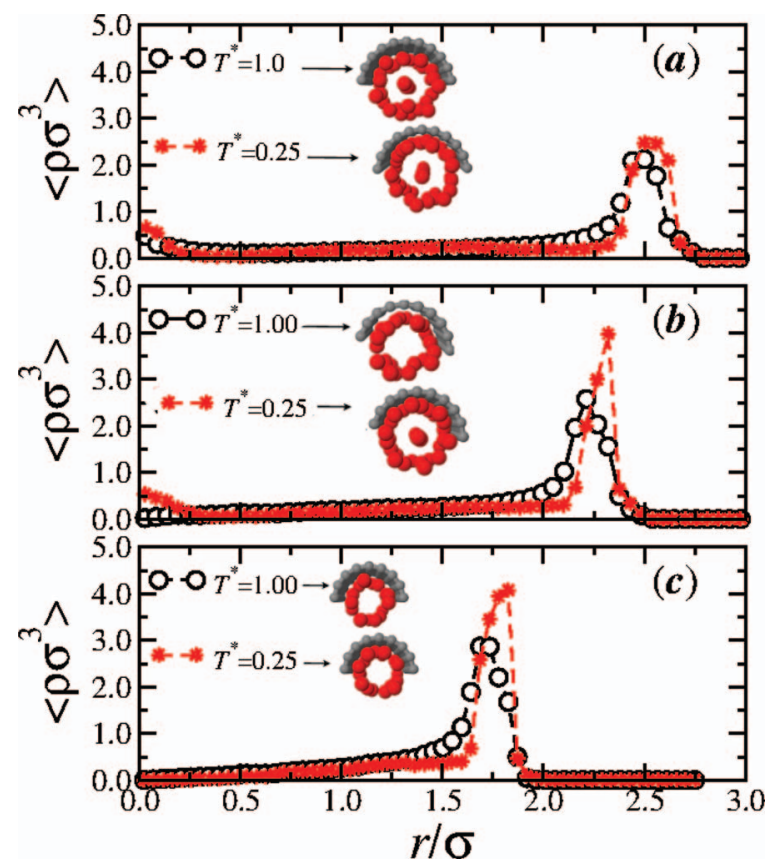

FIG. 7. Radial density profile inside the nanotube for $T^{*}=1.0$ (black circles) and $T^{*}=0.25$ (red stars), for radius (a) $a=3.5 \sigma$, (b) $a=3.25 \sigma$, and (c) $a=2.75 \sigma$. The snapshots show the different observed structures. 
form structures that increase the enthalpic contribution to the free energy, minimizing it. On the other hand, the confinement imposes restrictions to ordering, increasing the entropic contribution to the free energy. As a consequence, the transition from the structure with two layers to one single cylindrical layer occurs first in systems at higher temperature, or higher entropic contribution. For sufficiently low values of the nanotube radius, the flowing system is characterized by a single cylindrical layer regardless of the temperature, which is the case for $a=2.75 \sigma$, as shown in Fig. 7(c).

\section{CONCLUSION}

In summary, we have studied the effect of confinement in the flow of particles interacting through core-softened potentials. In the presence of two competing length scales our results show an anomalous oscillatory behavior, in the form of a global minimum flux with the nanotube radius, which is compatible with a transition from single-file flow to the flow of an ordered-like fluid. At increased values of the radius, a local minimum in the overall mass flux appears, which is more evident at low temperatures, and reflects the formation of a double-layer of flowing particles through the nanotube. These anomalous flow properties of core-softened particles in confined cylindrical geometries provide useful insight to the microscopical description of the flow behavior of nanoconfined anomalous liquids.

\section{ACKNOWLEDGMENTS}

We acknowledge financial support from the Brazilian Agencies CNPq, CAPES, FAPERGS, INCT-FCx, and FUNCAP, the FUNCAP/CNPq Pronex grant, and the National Institute of Science and Technology for Complex Systems in Brazil.

${ }^{1}$ M. W. J. Prins, W. J. J. Welters, and J. W. Weekamp, Science 291, 277 (2001).

${ }^{2}$ S. Hohmann, S. Nielsen, and P. Agre, Current Topics in Membranes: Aquaporins (Academic Press, San Diego, 2001).

${ }^{3}$ M. W. J. Prins, W. J. J. Welters, and J. W. Weekamp, Nano Lett. 12, 5740 (2012).

${ }^{4}$ G. S. Kell, J. Chem. Eng. Data 20, 97 (1975).

${ }^{5}$ C. A. Angell, E. D. Finch, and P. Bach, J. Chem. Phys. 65, 3063 (1976).

${ }^{6}$ F. X. Prielmeier, E. W. Lang, R. J. Speedy, and H.-D. Lüdemann, Phys. Rev. Lett. 59, 1128 (1987).

${ }^{7}$ H. Thurn and J. Ruska, J. Non-Cryst. Solids 22, 331 (1976).

${ }^{8}$ Handbook of Chemistry and Physics, 65th ed. (CRC Press, Boca Raton, FL, 1984).

${ }^{9}$ G. E. Sauer and L. B. Borst, Science 158, 1567 (1967).

${ }^{10}$ S. J. Kennedy and J. C. Wheeler, J. Chem. Phys. 78, 1523 (1983).

${ }^{11}$ T. Tsuchiya, J. Phys. Soc. Jpn. 60, 227 (1991).

${ }^{12}$ P. T. Cummings and G. Stell, Mol. Phys. 43, 1267 (1981).

${ }^{13}$ M. Togaya, Phys. Rev. Lett. 79, 2474 (1997).

${ }^{14}$ C. A. Angell, R. D. Bressel, M. Hemmatti, E. J. Sare, and J. C. Tucker, Phys. Chem. Chem. Phys. 2, 1559 (2000).

${ }^{15}$ M. S. Shell, P. G. Debenedetti, and A. Z. Panagiotopoulos, Phys. Rev. E 66, $011202(2002)$

${ }^{16}$ R. Sharma, S. N. Chakraborty, and C. Chakravarty, J. Chem. Phys. 125, 204501 (2006).
${ }^{17}$ S. Sastry and C. A. Angell, Nat. Mater. 2, 739 (2003).

${ }^{18}$ S.-H. Chen, F. Mallamace, C.-Y. Mou, M. Broccio, C. Corsaro, A. Faraone, and L. Liu, Proc. Natl. Acad. Sci. U.S.A. 103, 12974 (2006).

${ }^{19}$ T. Morishita, Phys. Rev. E 72, 021201 (2005).

${ }^{20}$ P. A. Netz, F. W. Starr, H. E. Stanley, and M. C. Barbosa, J. Chem. Phys. 115, 344 (2001).

${ }^{21}$ P. A. Netz, F. W. Starr, M. C. Barbosa, and H. E. Stanley, Physica A 314, 470 (2002).

${ }^{22}$ M. Majumder, N. Chopra, R. Andrews, and B. J. Hinds, Nature (London) 438, 4 (2005).

${ }^{23}$ J. K. Holt, H. G. Park, Y. M. Wang, M. Stadermann, A. B. Artyukhin, C. P. Grigoropulos, A. Noy, and O. Bakajin, Science 312, 1034 (2006).

${ }^{24}$ M. Whitby, L. Cagnon, M. Thanou, and N. Quirke, Nano Lett. 8, 2632 (2008).

${ }^{25}$ X. Qin, Q. Yuan, Y. Zhao, S. Xie, and Z. Liu, Nano Lett. 11, 2173 (2011).

${ }^{26}$ A. I. Kolesnikov, J. M. Zanotti, C. K. Long, P. Thiygarajan, A. P. Moravsky, R. O. Loutfy, and C. J. Burnham, Phys. Rev. Lett. 93, 035503 (2004).

${ }^{27}$ J. A. Thomas and A. J. H. Macgaughey, Phys. Rev. Lett. 102, 184502 (2009).

${ }^{28}$ K. P. Lee, H. Leese, and D. Mattia, Nanoscale 4, 2621 (2012).

${ }^{29}$ K. Falk, F. Sedlmeier, L. Joly, R. R. Netz, and L. Bocquet, Nano Lett. 10, 4067 (2010).

${ }^{30}$ S. D. Kannan, B. D. Todd, J. S. Hansen, and P. J. Davies, J. Chem. Phys. 136, 024705 (2012).

${ }^{31}$ S. D. Kannan, B. D. Todd, J. S. Hansen, and P. J. Davies, J. Chem. Phys. 138, 094701 (2013).

${ }^{32}$ G. Malescio, G. Franzese, A. Skibinsky, S. V. Buldyrev, and H. E. Stanley, Phys. Rev. E 71, 061504 (2005).

${ }^{33}$ E. A. Jagla, Phys. Rev. E 58, 1478 (1998).

${ }^{34}$ A. Scala, F. W. Starr, E. La Nave, F. Sciortino, and H. E. Stanley, Nature (London) 406, 166 (2000).

${ }^{35}$ L. Xu, P. Kumar, S. V. Buldyrev, S.-H. Chen, P. Poole, F. Sciortino, and H. E. Stanley, Proc. Natl. Acad. Sci. U.S.A. 102, 16558 (2005).

${ }^{36}$ A. B. de Oliveira, P. A. Netz, T. Colla, and M. C. Barbosa, J. Chem. Phys. 124, 084505 (2006).

${ }^{37}$ P. H. Poole, F. Sciortino, U. Essmann, and H. E. Stanley, Nature (London) 360, 324 (1992).

${ }^{38}$ A. B. de Oliveira, P. A. Netz, T. Colla, and M. C. Barbosa, J. Chem. Phys. 125, 124503 (2006).

${ }^{39}$ N. M. Barraz, Jr., E. Salcedo, and M. C. Barbosa, J. Chem. Phys. 131, 094504 (2009).

${ }^{40}$ J. N. da Silva, E. Salcedo, A. B. de Oliveira, and M. C. Barbosa, J. Chem. Phys. 133, 244506 (2010).

${ }^{41}$ A. H. Marcus and S. A. Rice, Phys. Rev. E 55, 637 (1997).

${ }^{42}$ H. Löwen, Physica A 235, 129 (1997).

${ }^{43}$ L. Krott and M. C. Barbosa, J. Chem. Phys. 138, 084505 (2013).

${ }^{44}$ L. Krott and J. R. Bordin, J. Chem. Phys. 139, 154502 (2013).

${ }^{45}$ L. Krott and M. C. Barbosa, Phys. Rev. E 89, 012110 (2014).

${ }^{46}$ J. R. Bordin, A. B. de Oliveira, A. Diehl, and M. C. Barbosa, J. Chem. Phys 137, 084504 (2012).

${ }^{47}$ J. R. Bordin, A. Diehl, and M. C. Barbosa, J. Phys. Chem. B 117, 7047 (2013).

${ }^{48}$ F. de los Santos and G. Franzese, Phys. Rev. E 85, 010602 (2012).

${ }^{49}$ J. R. Bordin, A. Diehl, M. C. Barbosa, and Y. Levin, Phys. Rev. E 85, 031914 (2012).

${ }^{50}$ S. Berbardi, B. D. Todd, and D. J. Searles, J. Chem. Phys. 132, 244706 (2010).

${ }^{51}$ X. Yong and L. T. Zhang, J. Chem. Phys. 138, 084503 (2013).

${ }^{52}$ J. R. Bordin, L. B. Krott, and M. C. Barbosa, J. Phys. Chem. C 118, 9497 (2014).

${ }^{53}$ P. Allen and D. J. Tildesley, Computer Simulation of Liquids (Oxford University Press, Oxford, 1987).

${ }^{54}$ A. B. de Oliveira, E. Salcedo, C. Chakravarty, and M. C. Barbosa, J. Chem. Phys. 132, 234509 (2010).

${ }^{55}$ G. S. Kell, J. Chem. Eng. Data 12, 66 (1967).

${ }^{56}$ G. S. Heffelfinger and F. Van Smol, J. Chem. Phys. 100, 7548 (1994).

${ }^{57}$ Y. Zheng, H. Ye, Z. Zhang, and H. Zhang, Phys. Chem. Chem. Phys. 14, 964 (2012).

${ }^{58}$ A. Striolo, Nano Lett. 6, 633 (2006). 